\title{
Avoiding stair-step artifacts in image registration for GOES-R navigation and registration assessment
}

\section{(A) AEROSPACE

Thomas J. Grycewicz*a, Frank J. De Lucciaa ${ }^{\mathrm{a}}$ Bin Tan ${ }^{\mathrm{b}, \mathrm{d}}$ Peter J Isaacson ${ }^{\mathrm{a}}$, John Dellomo ${ }^{\mathrm{c}, \mathrm{d}}$

aThe Aerospace Corporation, 2310 E. El Segundo BI., El Segundo, CA USA 90245-4691, bSSAI, 10210 Greenbelt Rd., Suite 600, Lanham, MD USA 20706, 'GST, 7855 Walker dr., Suite 200, Greenbelt, MD USA 20770,

dNASA GSFC Terrestrial Information Systems Laboratory,8800 Greenbelt RD., Greenbelt, MD 20771

SPIE Optical Engineering and Applications 28 August - 1 September 2016

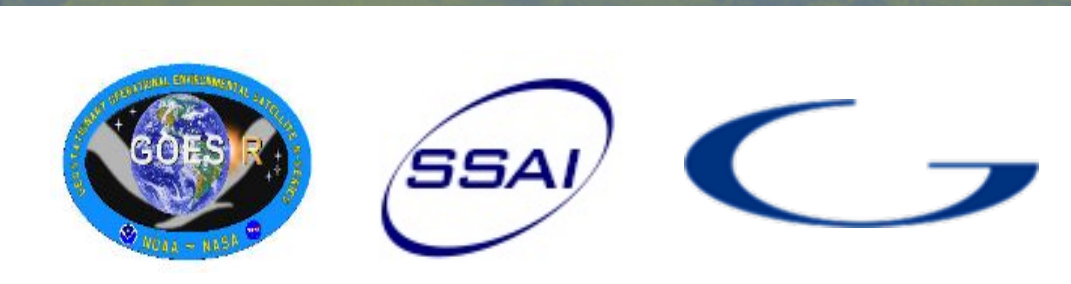




\section{Overview}

- Stair-step is a subpixel image registration artifact

- We have seen this before... ten years ago

- GOES-R Advanced Baseline Imager (ABI) Instrument Navigation and Registration (INR) assessment

- Methods to reduce the stair-step artifact

- Conclusions 


\section{Shift Estimation using Image Registration}
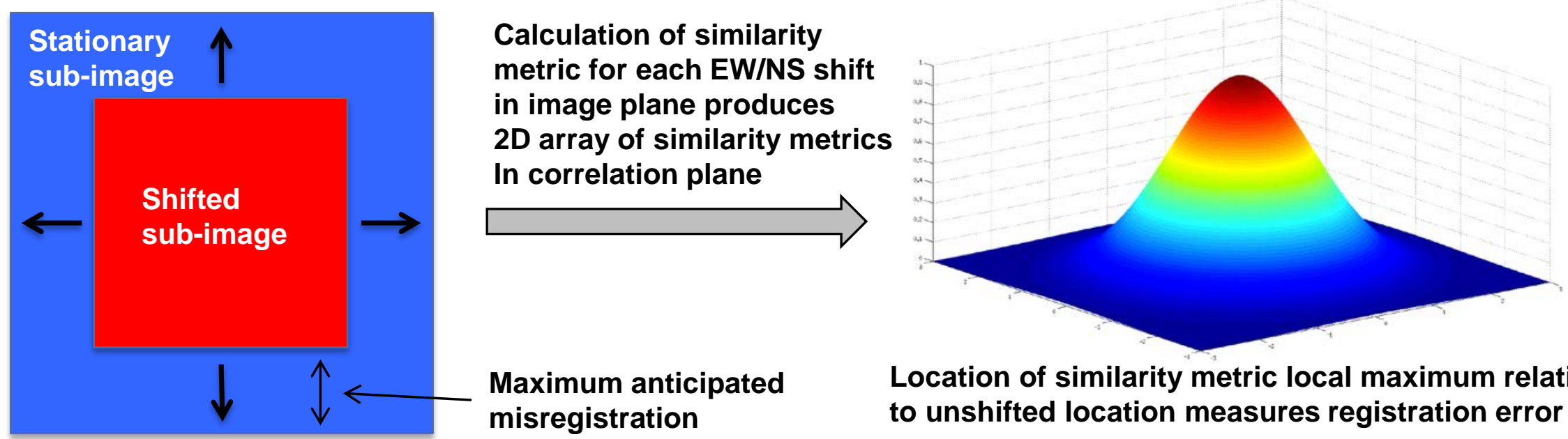

Location of similarity metric local maximum relative to unshifted location measures registration error

- Similarity metric uses a form of image correlation

- Normalized cross-correlation (Pearson coefficient)

- Normalized Mutual Information (NMI)

- Phase Correlator (Fourier processing)

- Output increases as images are shifted towards perfect alignment

- Inputs are pixelated with same pixel size

- Output is similarly pixelated

- Maximum location shows image shift to nearest pixel

- Interpolation is required to estimate sub-pixel registration

- Stair-step is an interpolation artifact

Common concept for measuring GOES-R INR metrics 


\section{Characteristics of the Stair-Step Artifact}

\section{Subtitle}

True

Displacement

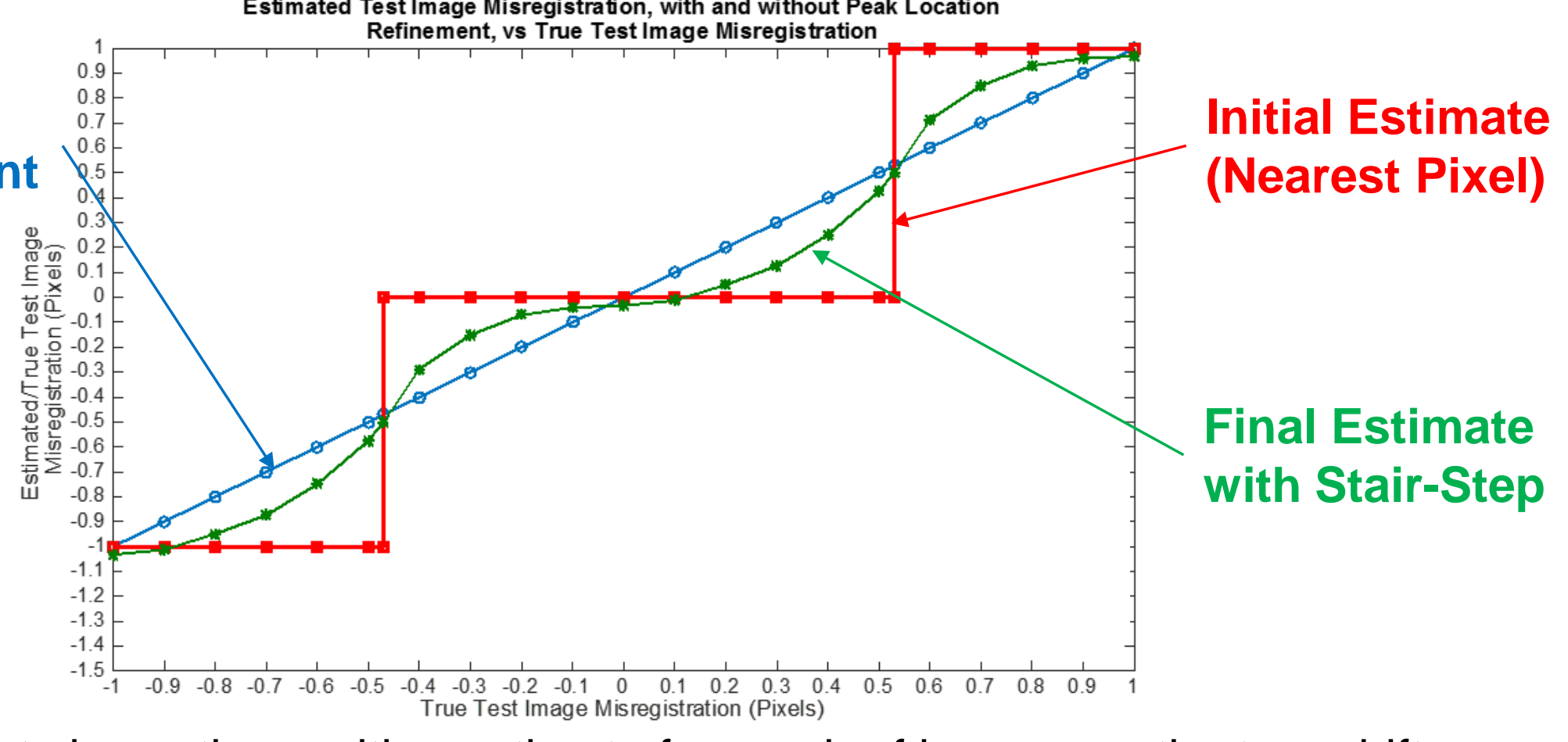

- This chart shows the position estimate for a pair of images as the true shift varies from -1 to 1 pixel

- The initial estimate of registration is the nearest-pixel location

- We fit the correlation peak with a quadratic for the final estimate

- Correct for offsets of an integer number of pixels

Stair-step is a sub-pixel estimation artifact 


\section{Characteristics of the Stair-Step Artifact}

\section{Subtitle}

Test Image Misregistration, with and without Peak Location

True

Displacement

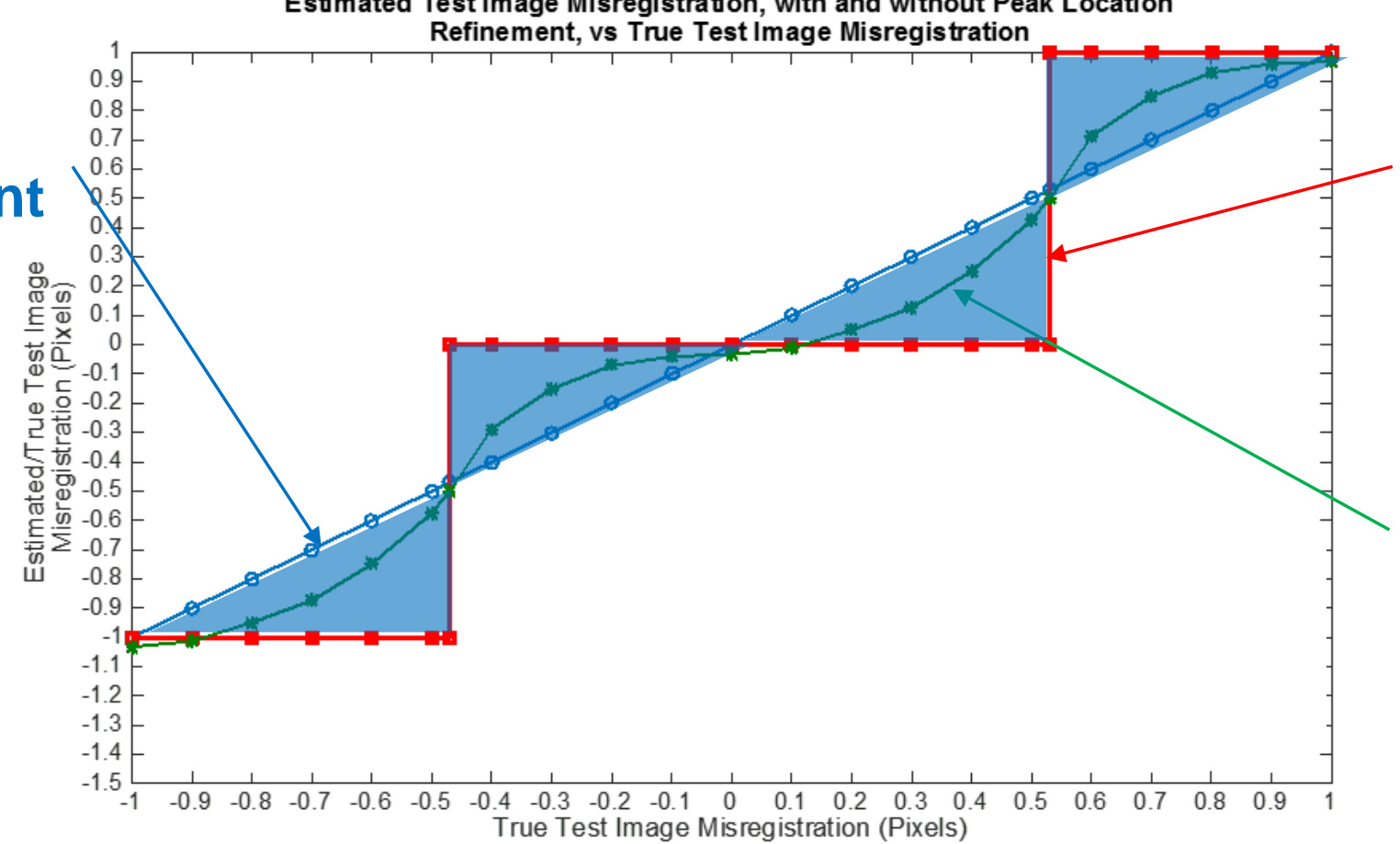

Initial Estimate (Nearest Pixel)

Final Estimate with Stair-Step

- We fit the correlation peak with a quadratic for the final estimate

- Correct for offsets of an integer number of pixels

- Subpixel shifts require interpolation which can introduce stair-step

- Estimates with stair-step fall between the true location and the nearest neighbor in the blue zone

Stair-step is a sub-pixel estimation artifact 


\section{Ten Years Ago....}

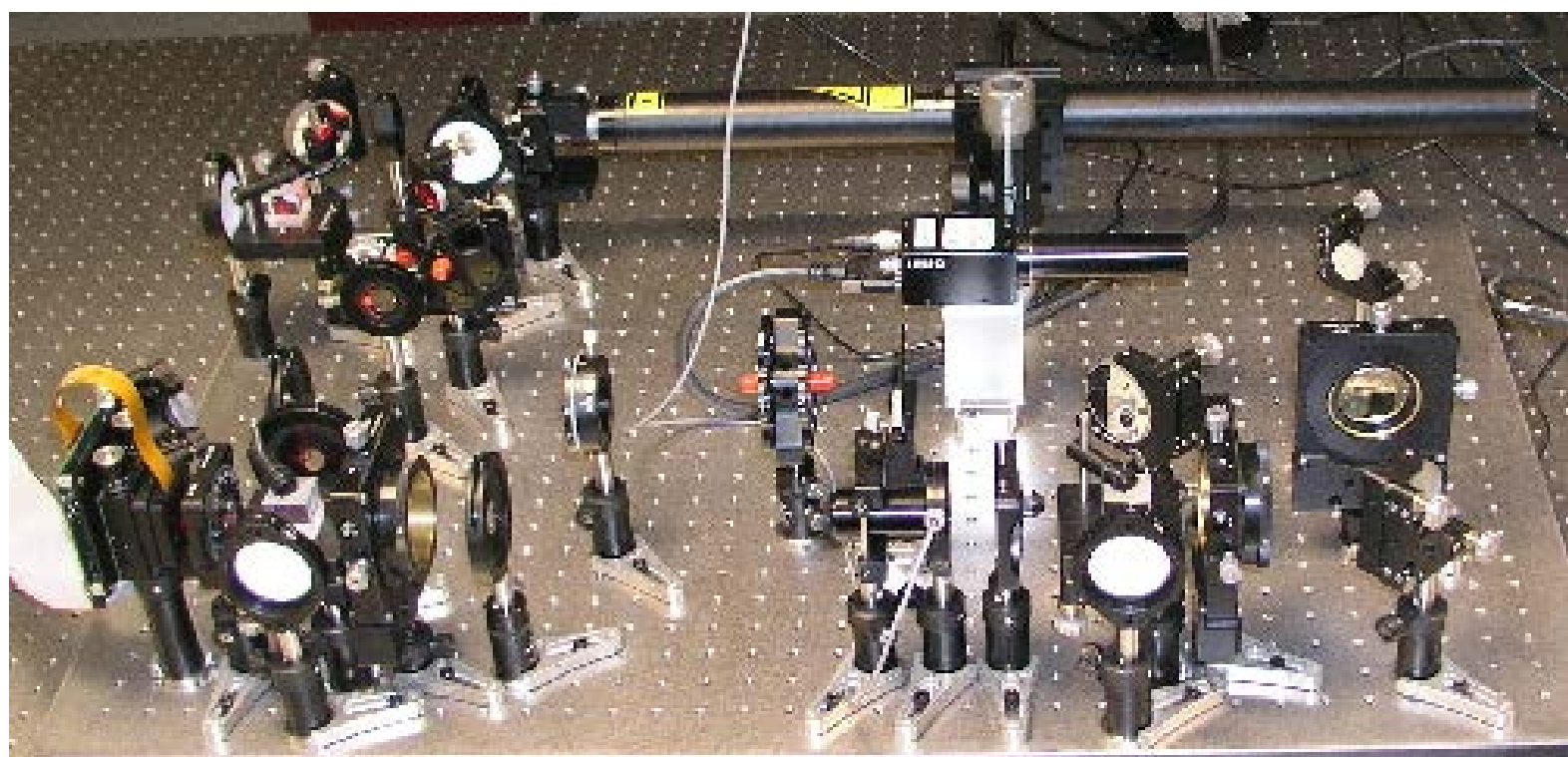

Third Generation (all optical) Correlator

SS Nanpert

Operated in burst mode (5-150 frames) Input camera operates at 400 FPS Input pre-processing at 50 FPS Optical correlator operates at 50 FPS (Hamamatsu OASLM has $\sim 12 \mathrm{~ms}$ response time)

Correlation location calculated 50 FPS Output interpretation takes minutes

Material from Grycewicz et.al., Proc. SPIE 66950J

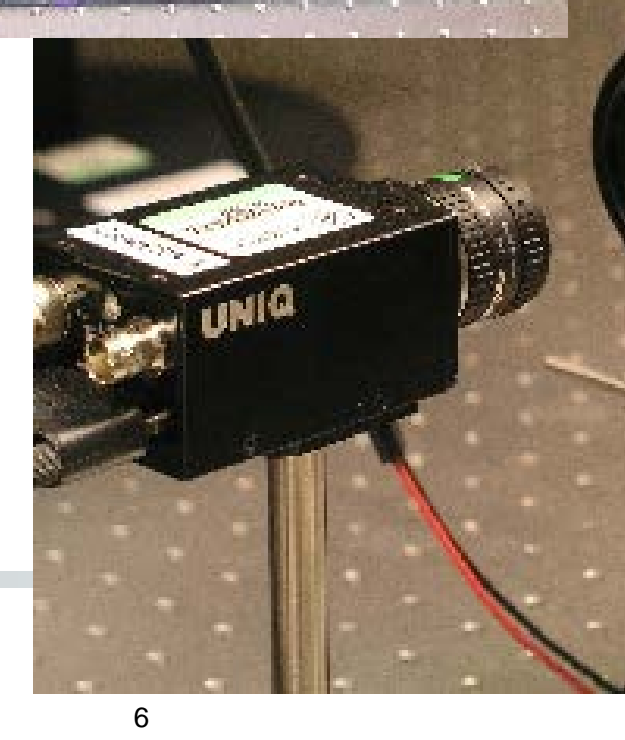

Spring 2006

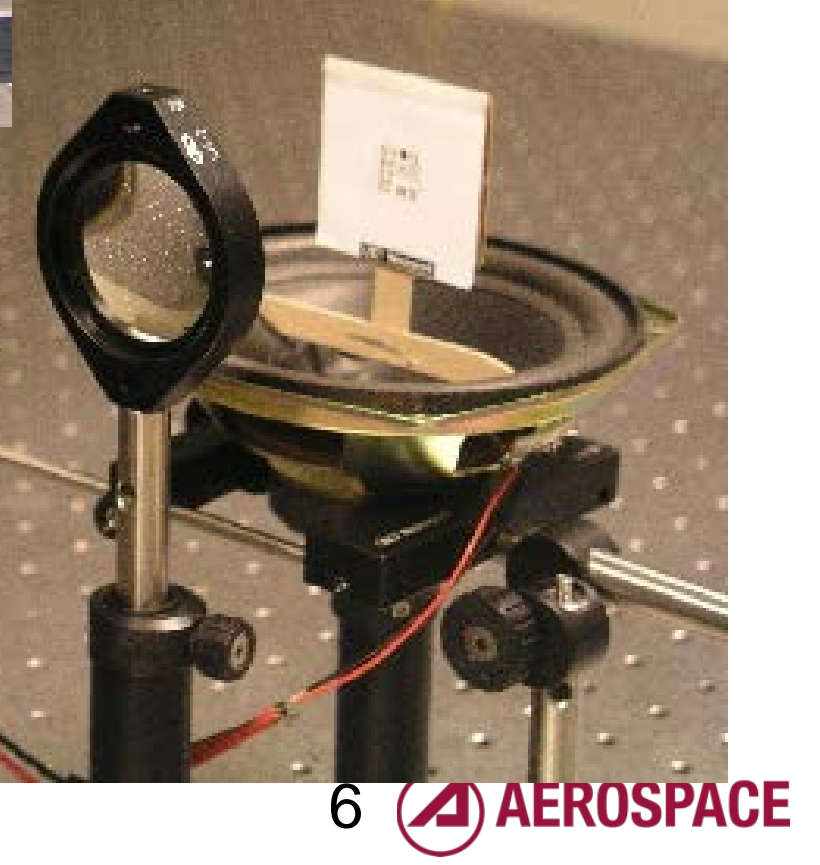




\section{All-Optical Correlator Registration Results}

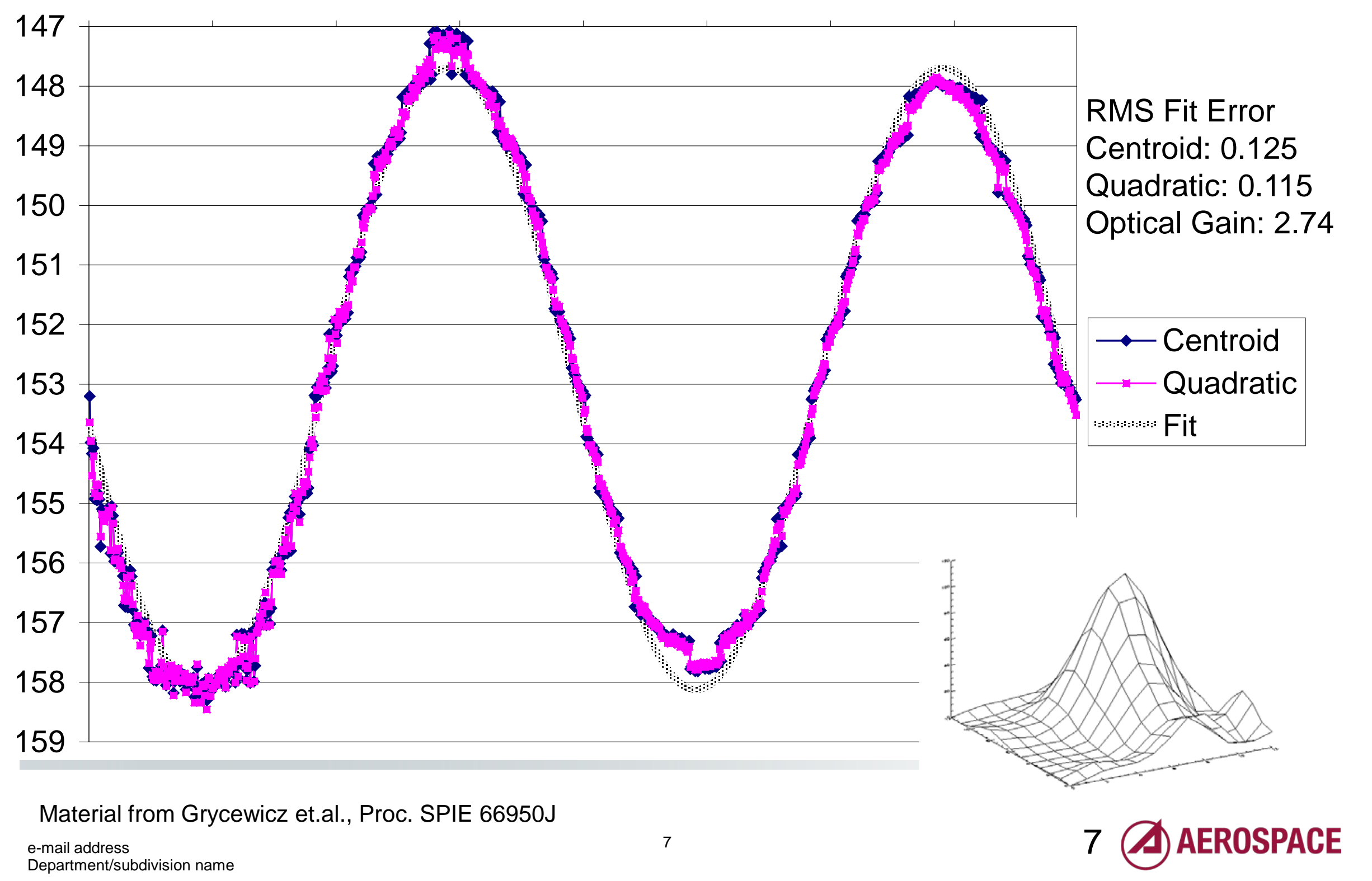




\section{Stair-Step is Hard to Detect}

\section{A sub-pixel artifact}

- Stair-step is a sub-pixel artifact

- It won't be seen except in an experiment with sub-pixel image displacement and sub-pixel ground truth

- Typically small-on order tenth pixel

- Registration is typically a point estimate

- You don't typically have a line of motion to estimate

- One estimate-one error

- Error is a combination of many system noise terms, including stair-step

- In the ABI case, our goal is to detect and measure subpixel misregistrations

- If present, the effect of stair-step will be to make the $A B I$ images appear better registered than they actually are 


\section{GOES-R INR Assessment}

- In March 2014 the GOES-R flight project initiated two efforts to develop tools for independent evaluation of on-orbit Image Navigation and Registration (INR) performance

- The Product Monitor (PM), developed by the ground project, provides heritage capability for INR performance assessment

- An independently developed capability for INR performance assessment using different techniques for risk reduction

- INR Performance Assessment Tool Set (IPATS) has been developed to:

- Independently measure INR performance characteristics

- Generate image-level and multi-image-level statistics

- Provide data visualization capability

- Archive results

- Aerospace is the primary architect and developer of IPATS, with final development and test ongoing jointly with SSAI and GST 


\section{INR metrics of interest}

- Navigation (NAV) error

- Difference between location of pixel in data product and true location

- Frame-to-frame registration (FFR) error

- Relative navigation error of corresponding pixels of same band in consecutive images

- Within-frame registration (WIFR) error

- Difference between radial separation of two pixels on the FG and their true angular separation

- Swath-to-swath registration (SSR) error

- Relative navigation error of two neighboring pixels on opposite sides of image swath boundary

- Channel-to-channel registration (CCR) error

- Relative navigation error of corresponding pixels of different bands in the same frame

Material from De Luccia et.al., Proc. SPIE 988119

Key metric for any type of error is "3-sigma error", 99.73" percentile of distribution of error magnitudes over a 24 hour data collection period. 


\section{Simulation Methodology}

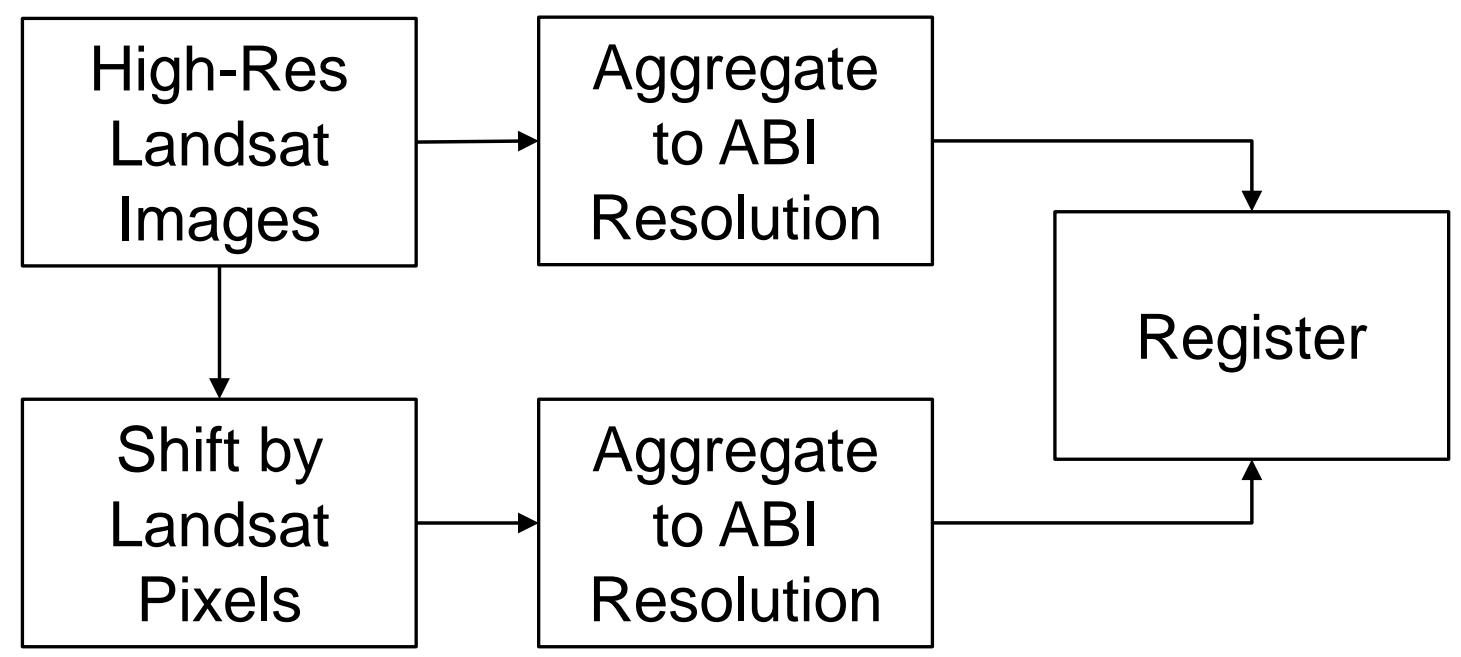

- Use IPATS tools and processes to register surrogate images

- Surrogate images have known ground truth

- Landsat images aggregated $25 \times 25$ or $33 \times 33$

- Registered images have GOES-like GSD of 750-1000 m

- Subpixel shifts in 0.03 or 0.04 pixel increments

- Used to simulate in-channel and channel-to-channel registration 


\section{Stair-Step in Surrogate GOES-R Images}

Simulations built with Landsat data
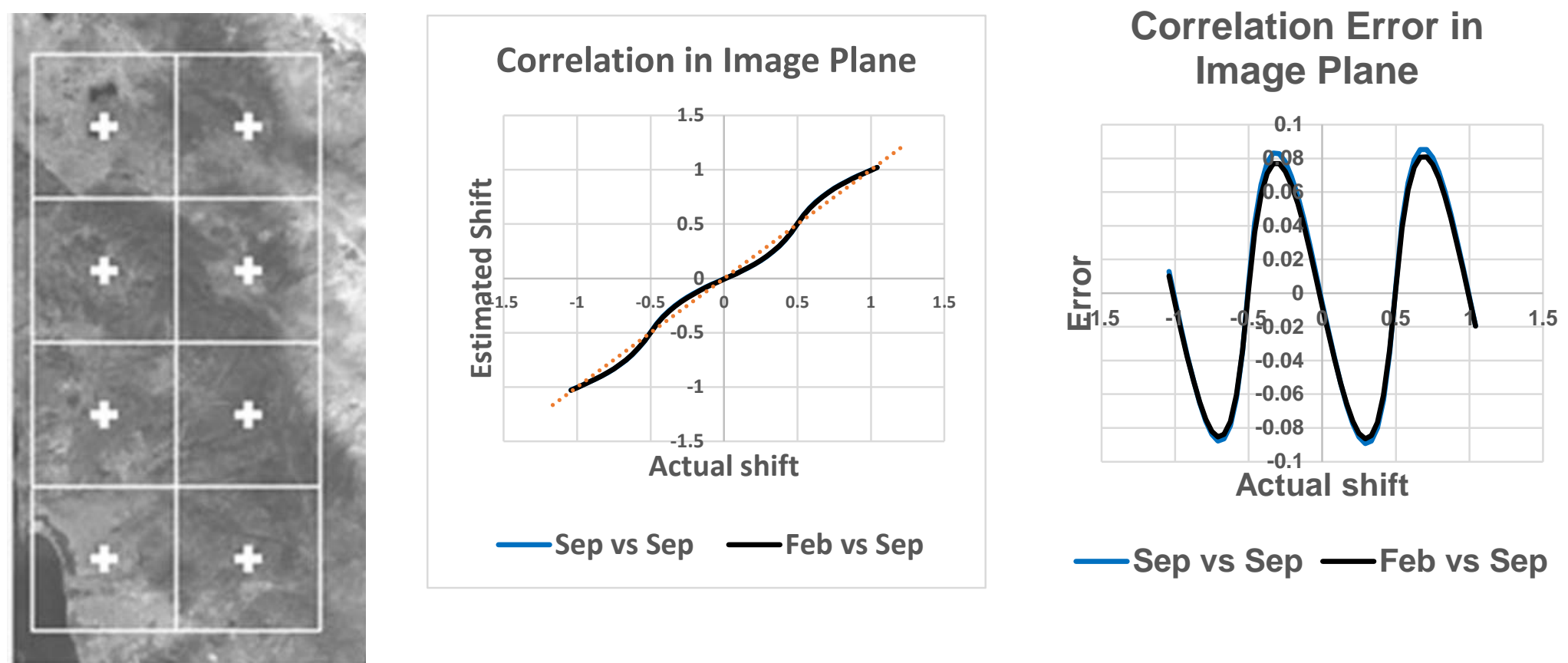

- Simulations use set of eight Landsat $64 \times 64$ pixel band 3 (red) chips

- Normalized cross-correlation (Pearson coefficient)

- Simulated motion by individual Landsat pixels (1/25 GOES pixel)

- RMS error 0.06 pixels

Stair-step seen in early simulations 


\section{Stair-Step Artifact in Simulated GOES-R Images}

- 30x30 pixel chip simulated from Landsat 7 band 3 (red)

Cross Correlation

$\operatorname{Max} 3 *$ STD $=0.160$

Min $3^{\star}$ STD $=0.070$

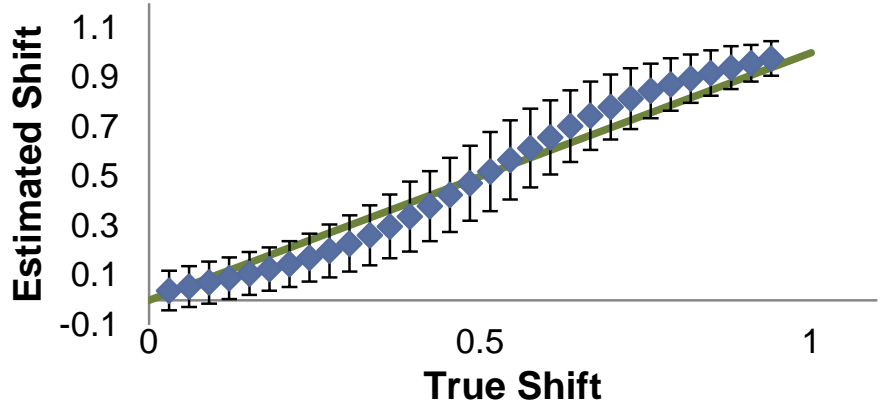

Normalized Mutual Information

- Set of 961 images

- Shifted by multiples of $1 / 33$ pixel

$\operatorname{Max} 3 * S T D=0.144$

Min $3^{*} \mathrm{STD}=0.055$

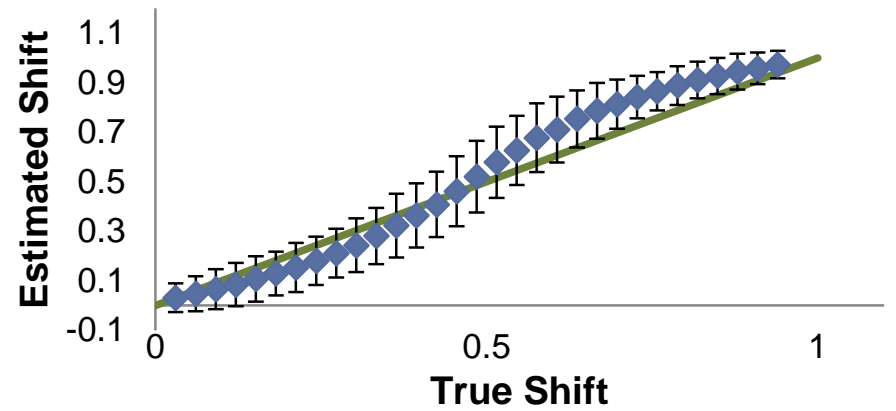

CC + Sobel

$\operatorname{Max} 3^{*} S T D=0.816$

Min $3^{*}$ STD $=0.023$

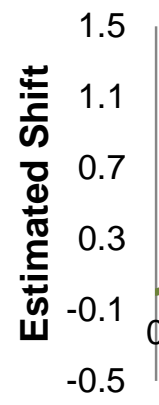

$-0.5$

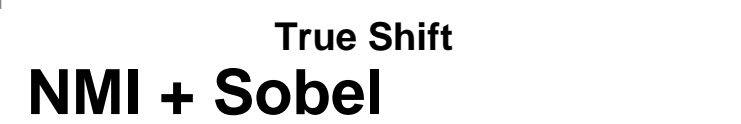

$\operatorname{Max} 3 *$ STD $=1.279$

Min $3^{*}$ STD $=0.017$

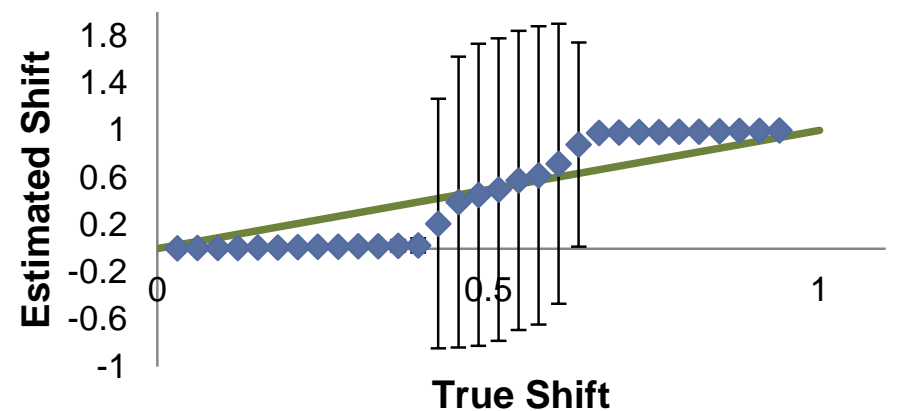

Stair-step is seen with many correlator types 


\section{Image Set Run in IPATS}

Simulation set used to characterize correlation techniques
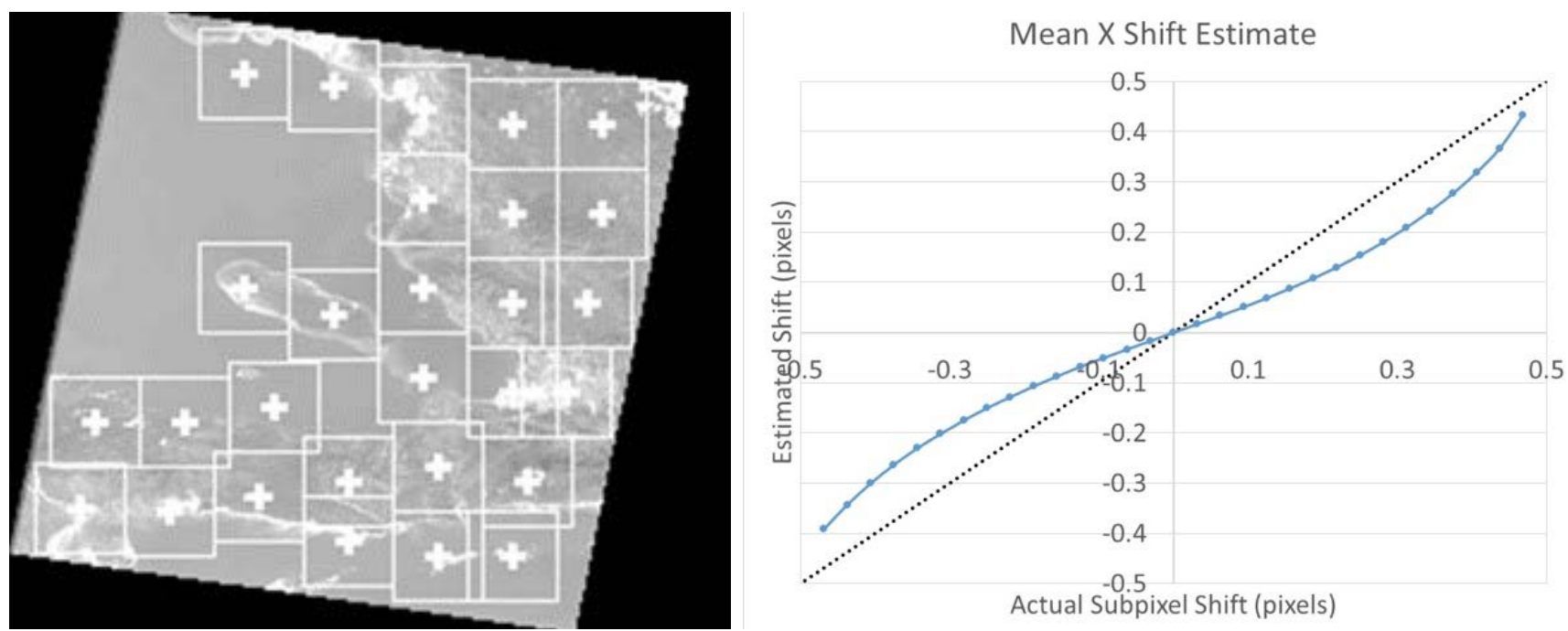

- This Landsat image of Haiti was used for simulations both within and outside of the IPATS framework

- The boxes define 30 pixel x 30 pixel $A B I$ correlation regions

- Visible and infrared bands were correlated 


\section{How can we reduce the size of the stair-step artifact?}

- Increase the resolution

- By estimating with high-resolution inputs, we get a high-resolution output

- Need to start with high-resolution inputs, otherwise its just interpolation

- Estimate the error and subtract

- If we can estimate the error, we can compensate

- A sinusoidal estimate works well for mild stair-step

- Choose a different correlator

- Different correlators have different stair-step response

- Choose a different output interpolator

- Stair-step is an interpolation artifact

- Choosing a good interpolator is critical 


\section{Increase Reference Resolution}

\section{Data from surrogate $A B I$ images on slide 12}

- Increasing the reference resolution results in a large reduction in the magnitude of stair-step

- Correlation done at the smaller pixel size

- The spatial period of the stair-step is reduced to the smaller pixel size

- This works well for NAV assessment

- Reference chips can have arbitrary scale

- Requires that one of inputs is available at high-resolution

- Not helpful for CCR or FFR

- When both images are rescaled, interpolating the inputs is similar to interpolating the output, but has computational disadvantages

\subsection{RMS}

\section{Correlation Error in Image Plane}

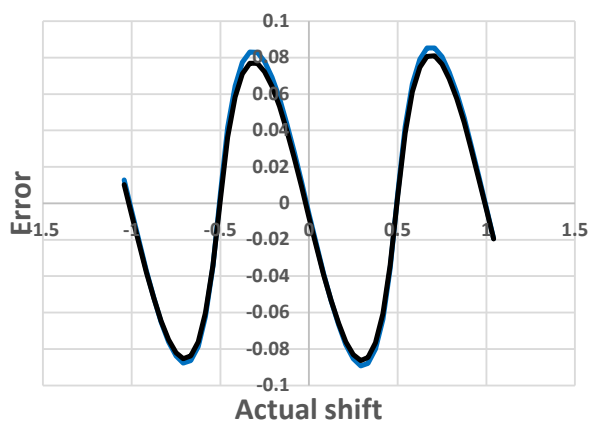

Correlation Error in Double Resolution Image Plane

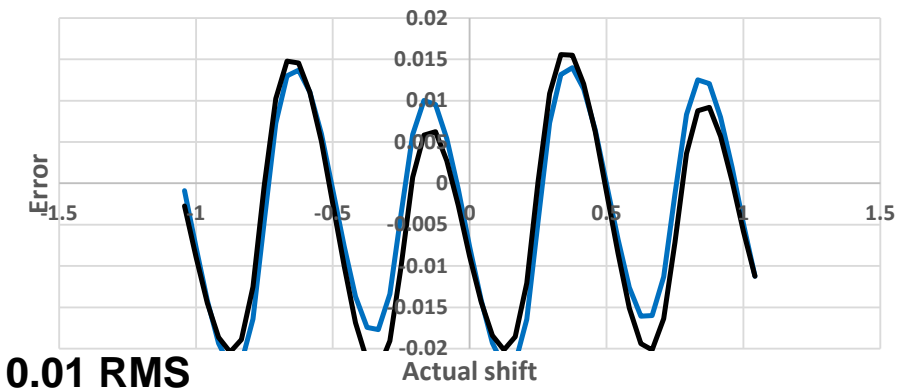

Correlation Error in Triple Resolution Image Plane

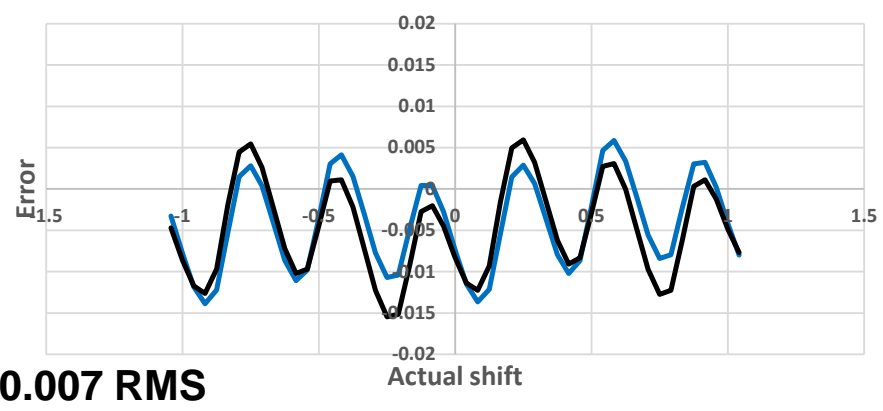

—Sep vs Sep —Feb vs Sep 


\section{Increasing Resolution by Interpolation of Both Inputs}

Data from surrogate $A B I$ images on slide 14

B2 vs B3 -- Pearson Coeficient

0.6

- Linear interpolation results in similar registration metrics at all zoom factors

- Here, SPF = Sub-Pixel Factor = amount of linear interpolation

- These correlations were all done within IPATS

- Very little difference is seen in the results

- "Truth" line has been adjusted for observed channel-to channel offset

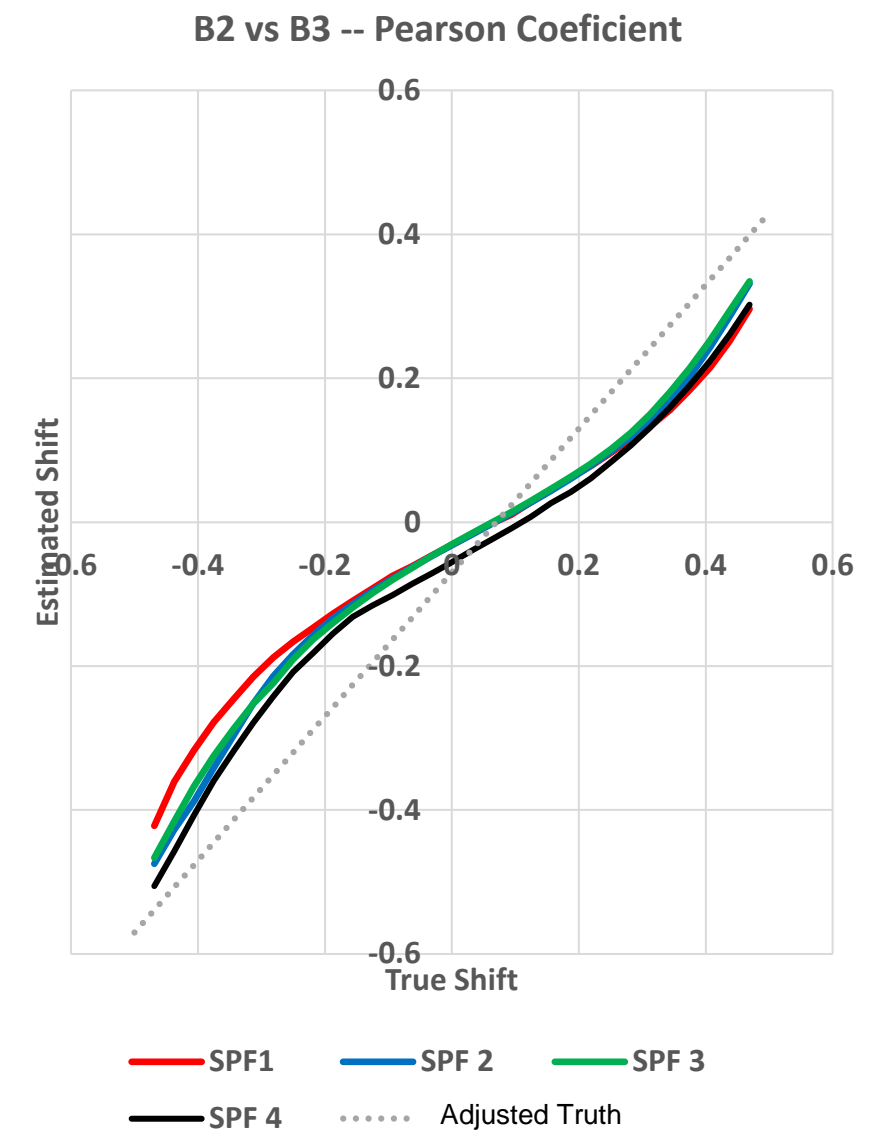




\section{Estimate the Error and Subtract}

Data from surrogate $A B I$ images on slide 12

- A small stair-case is estimated by a sinusoidal offset

- Zeroes at whole and half pixel shifts

- Maxima at $1 / 4$ and $3 / 4$ pixel shifts

- Magnitude can be estimated by modeling

- Stair-case cannot be eliminated this way, but can be greatly reduced

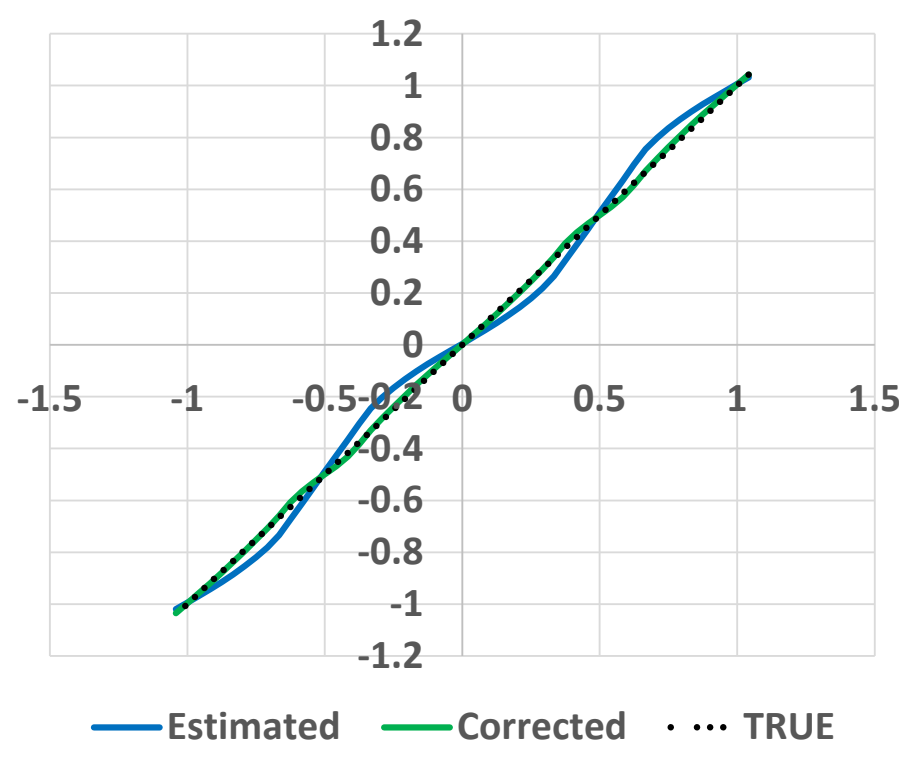

Error Estimate

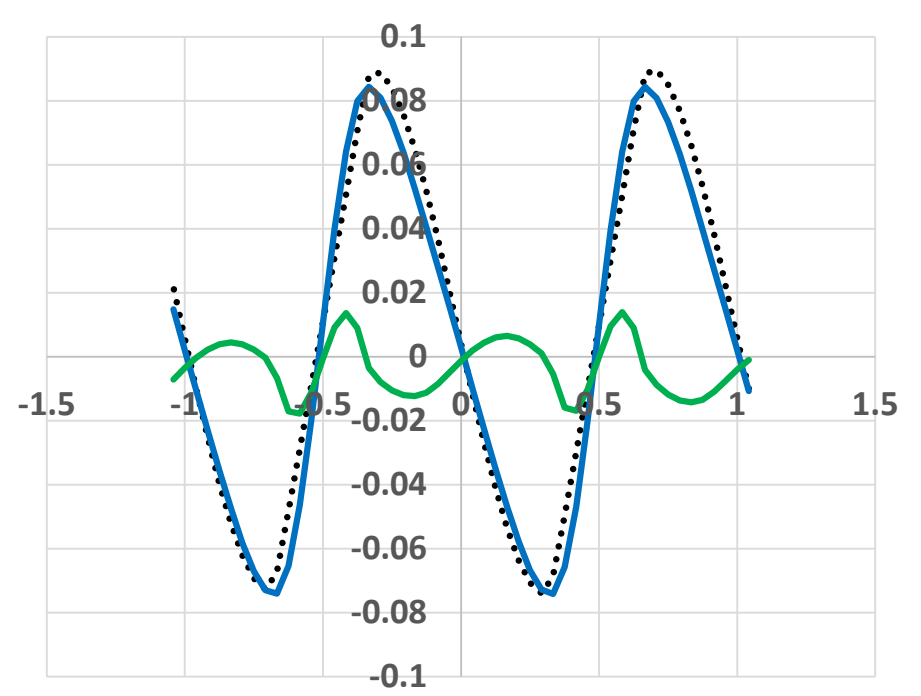
Corrected Error 


\section{Choose a Better Correlator}

B2 vs B3 with Shift

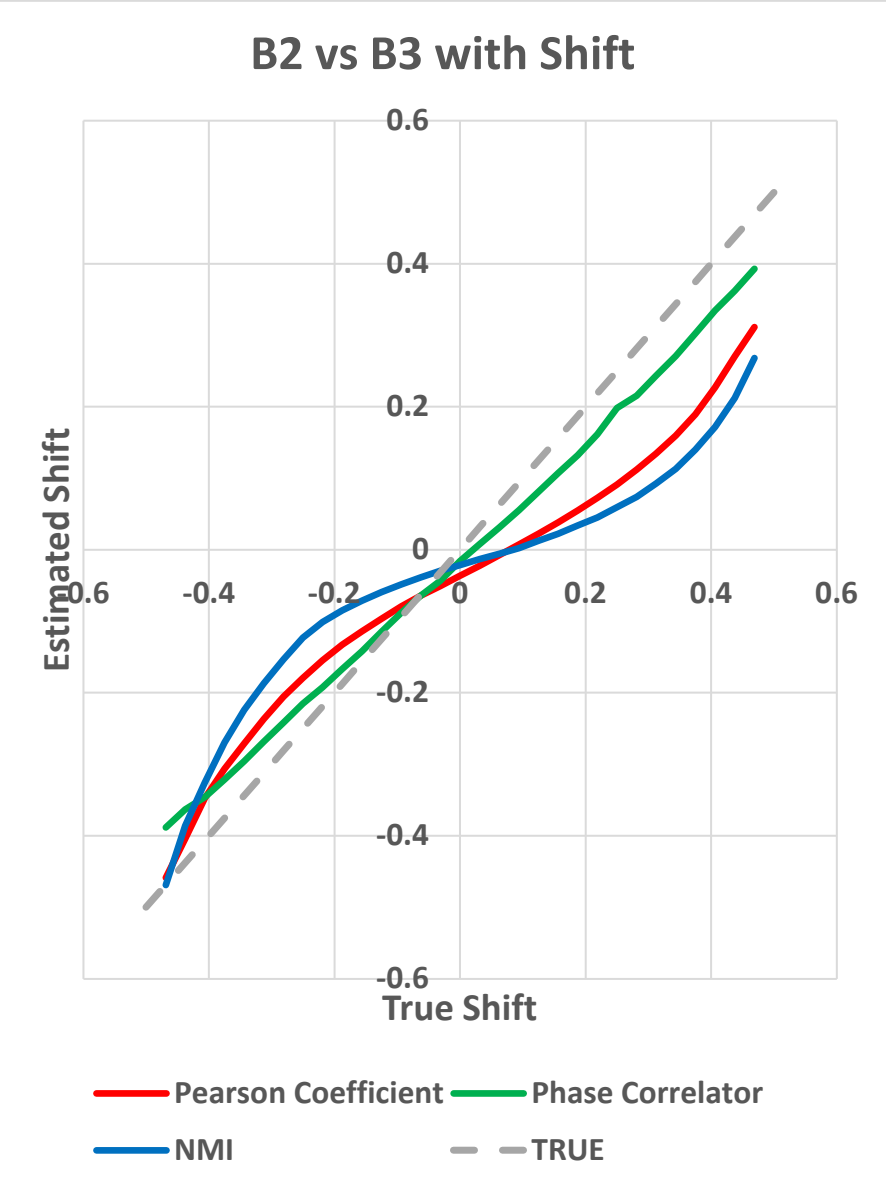

- Normalized Cross-Correlation (NCC) performs better than NMI when images are in similar bands

- NMI has an advantage for dissimilar CCR combinations-visible to IR

- A phase-space correlator operating in the Fourier domain is being evaluated as an alternate solution 


\section{Choose a Better Output Interpolator}

\section{Data from surrogate $A B I$ images on slide 12}

- The goal is to find the location of the primary peak in the output correlation plane at subpixel accuracy

- We have evaluated two interpolators

- Both interpolators start at the location defined by the largest value in the correlation plane

- The "Centroid" interpolator finds the center-of-mass of the pixels in a $5 \times 5$ region about the peak in the correlation plane

- The "Parabolic" interpolator fits onedimensional parabolas to the correlation peak in the $x$ and $y$ dimensions

B2 vs B3 -- Pearson Coeficient

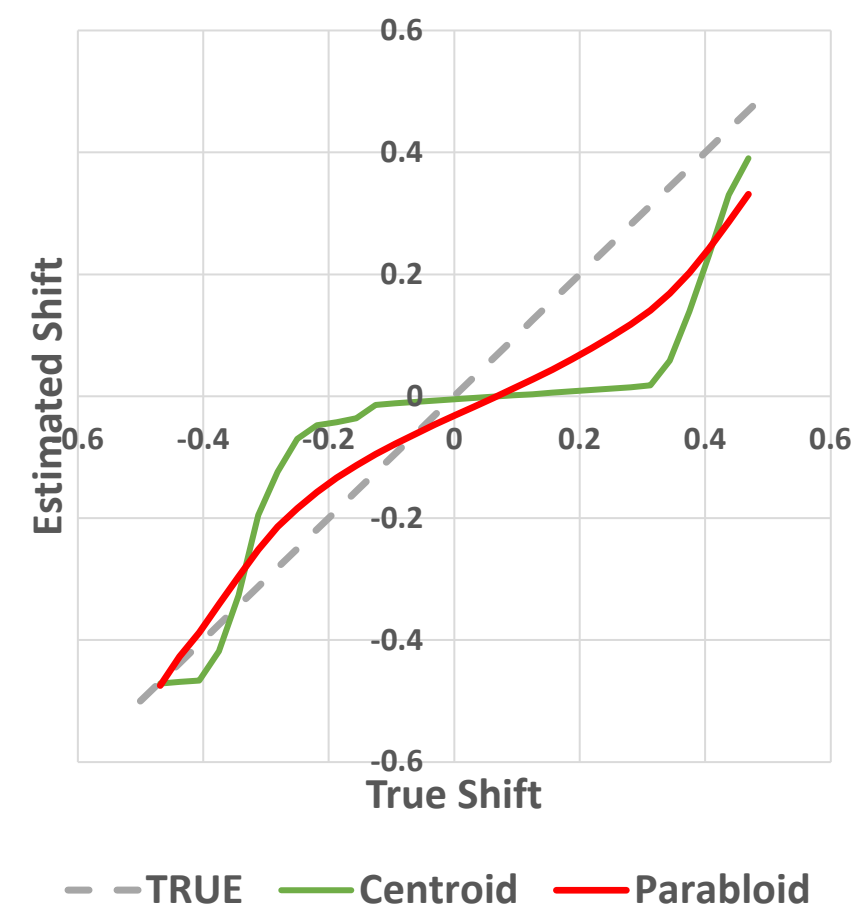




\section{Conclusions}

- We have described the stair-step registration artifact

- We have shown this may be an issue for GOES-R ABI registration

- Of order tenth pixel if misregistration is spread across a full pixel

- Much smaller if misregistration is always a quarter pixel or less

- Estimated subpixel misregistration smaller than actual

- Effect of stair-step can be minimized

- Good choice of correlator

- Good choice of interpolator

- Remaining effect can be estimated and compensated 


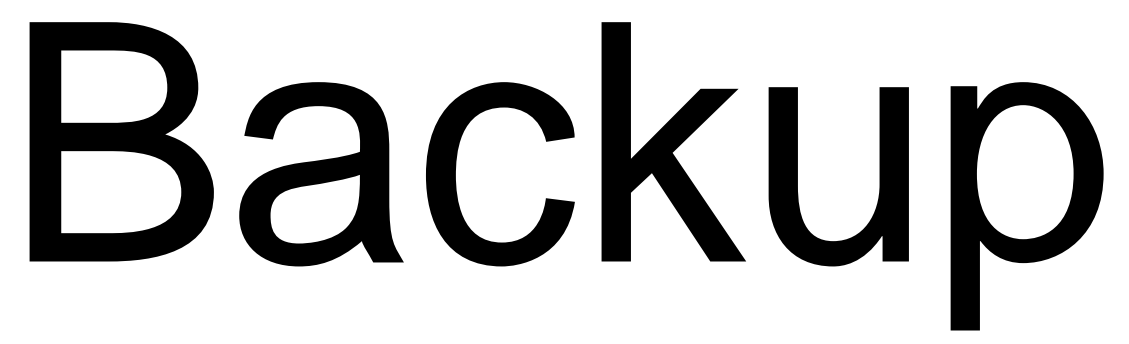




\section{But Why?}

\section{A Notional Explanation}

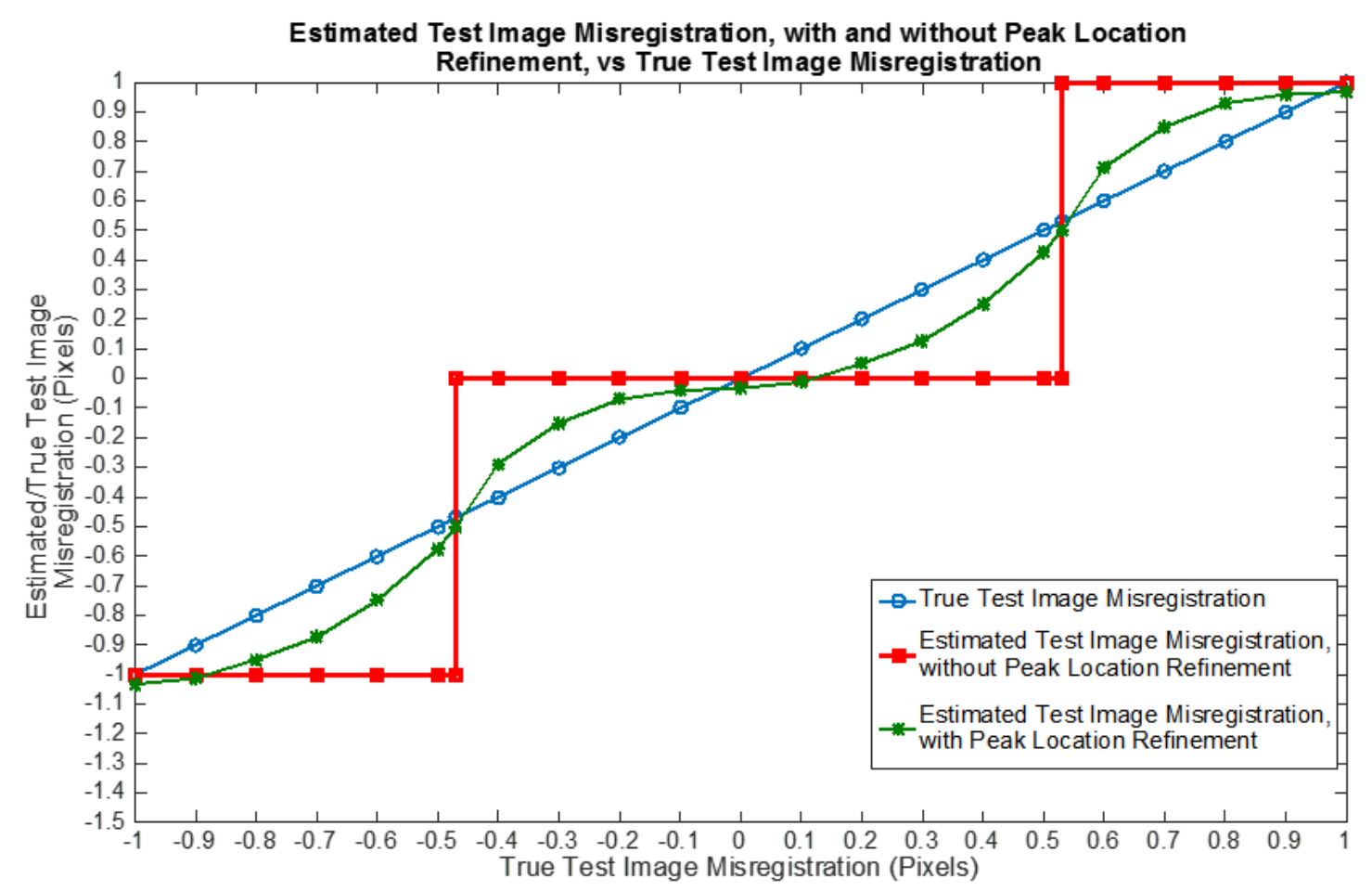

- I plan to build an animation here showing how the stair-step must arise if you start with an assumption that the measured values are correct in a region around the sample point

- That is, you get stair-step if you estimate the points but don't estimate or under-estimate the slopes

- Frank-I believe that the parabolic refinement will estimate the line with minimum slope that passes through three points. Is this true?

We assume an area around the measurement to be correct 


\section{Linear JTC Input and Correlation Peak}
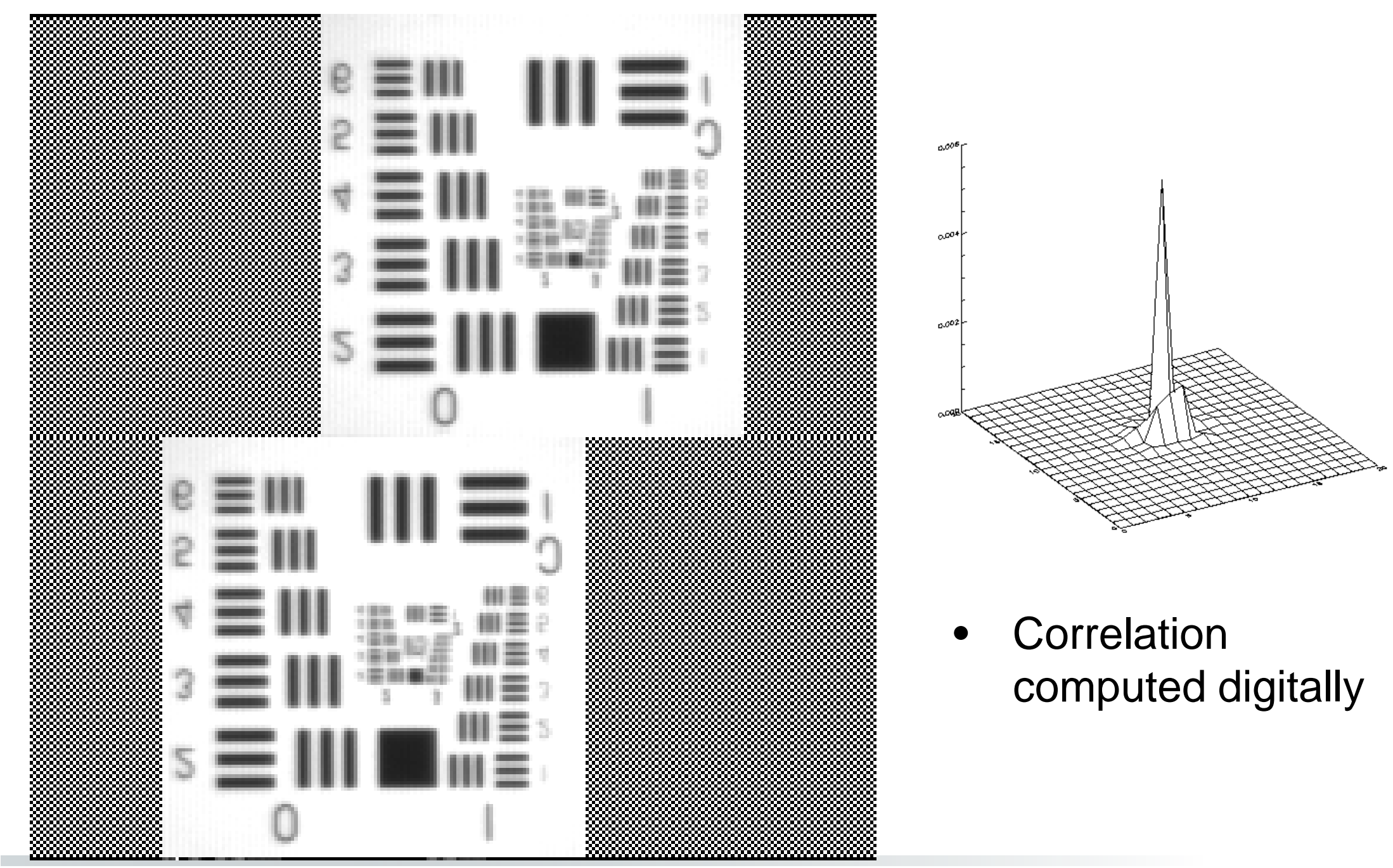

- Correlation computed digitally 


\section{Binary JTC Input and Correlation Peak}

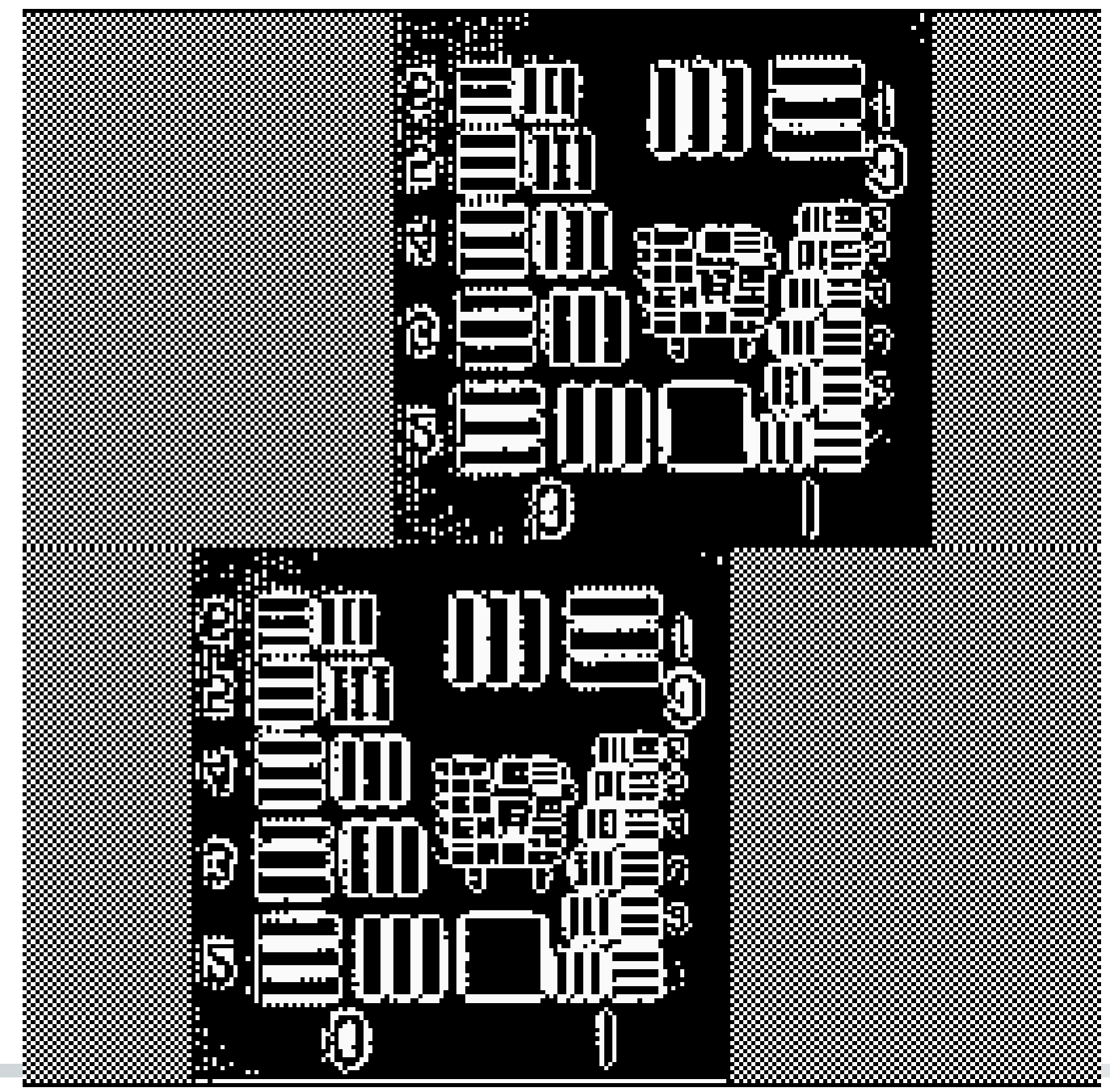

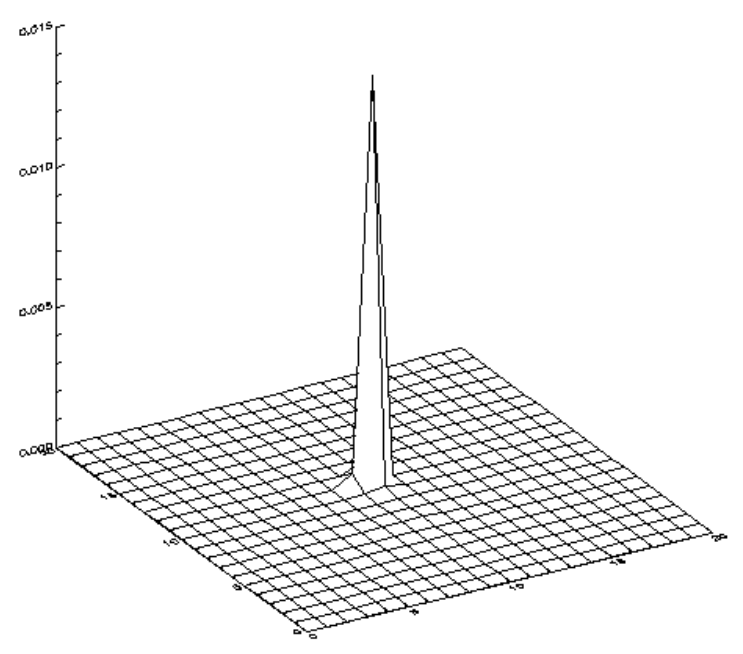

- Binarized by convolving with Laplacian kernel and thresholding

- Correlation computed digitally 


\section{Two-Stage Joint Transform Correlator, Binary Input}

\section{Two Stage BJTC}

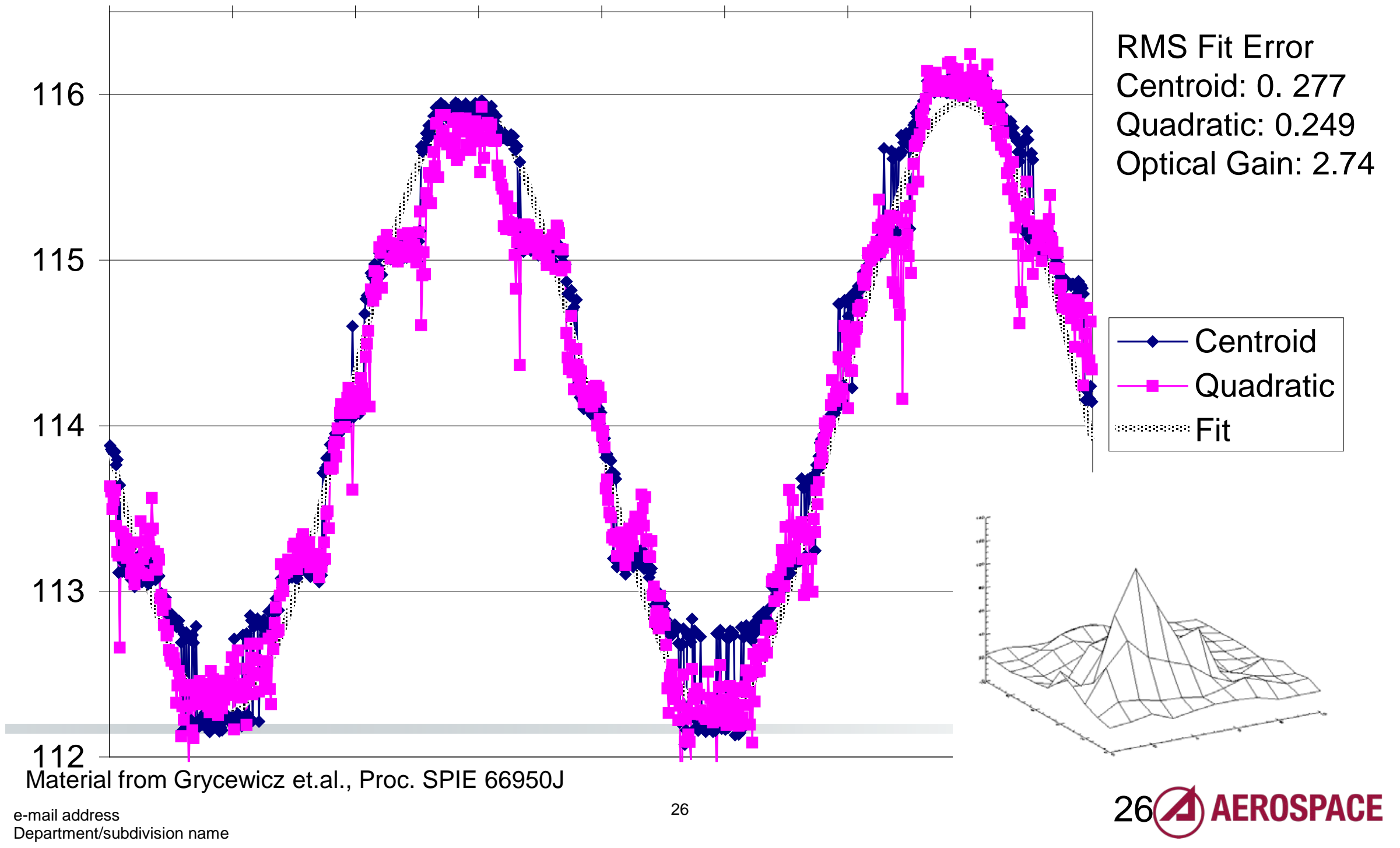




\section{All-Optical JTC, Linear Input}

\section{OASLM - Linear Input}

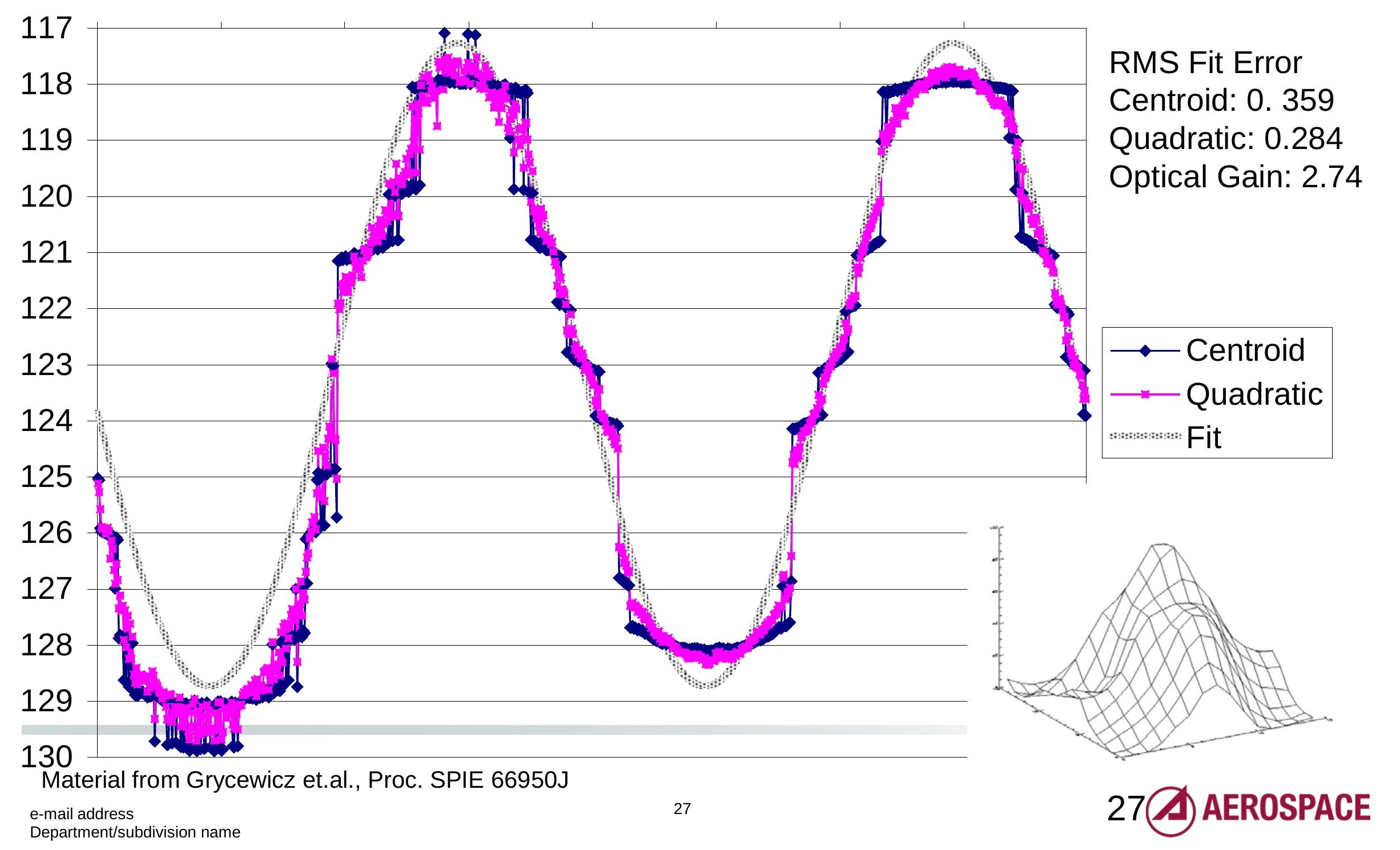

